\title{
Serum Ghrelin and Leptin Levels in Patients with Depression and the Effects of Treatment
}

\author{
Saliha Ozsoy ${ }^{1}$, Aslı Besirli², Ummuhan Abdulrezzak ${ }^{3}$, and Mustafa Basturk ${ }^{1}$ \\ ${ }^{1}$ Department of Psychiatry, Erciyes University School of Medicine, Kayseri, Turkey \\ ${ }^{2}$ Department of Psychiatry, Mus State Hospital, Mus, Turkey \\ ${ }^{3}$ Department of Nuclear Medicine, Erciyes University Medical School, Kayseri, Turkey
}

\begin{abstract}
Objective Ghrelin and leptin, appetite-regulating hormones, play a role in mood regulation. Current data about the relation between leptin/ghrelin and depression are still controversial. This study aimed to investigate serum leptin and ghrelin levels in patients with depression and the effects of treatment on these levels.

Methods Serum ghrelin and leptin levels were measured before and after treatment with antidepressant drugs and/or electroconvulsive therapy in 28 patients with depression and once in 21 healthy controls.

Results Serum ghrelin levels of the patients were high in the pre-treatment. After the treatment, ghrelin levels were not different from those of the controls. We found no difference in serum levels of leptin between the patients and controls and no change with treatment. body mass index of the patients increased after the treatment especially in the drug-treated group.

Conclusion The present study found increased serum ghrelin levels in depressive patients and normalization with improving of depression but no alteration in leptin levels.

Psychiatry Investig 2014;11(2):167-172
\end{abstract}

Key Words Depression, Ghrelin, Leptin, Treatment.

\section{INTRODUCTION}

The adipocyte-derived hormone leptin is known to regulate adipose tissue mass and energy balance and resistance to it is held responsible for obesity. ${ }^{1}$ There is increasing interest is in the role of this hormone in the regulation of mood. Animal studies showed that chronic stress decreased leptin level, leptin insufficiency was associated with depression-like behavior, and leptin had antidepressant-like efficacy. ${ }^{2}$ However, current information about the relation between leptin and human depression is still limited and controversial, despite studies on the subject for more than a decade. Human studies reported low ${ }^{3,4}$ or no difference ${ }^{5}$ in leptin levels in depressive patients. In a previous study, we found high leptin levels in

Received: April 20, 2013 Revised: September 5, 2013

Accepted: September 11, 2013 Available online: April 11, 2014

$\triangle$ Correspondence: Saliha Ozsoy, MD

Department of Psychiatry, Erciyes University School of Medicine, 38039 Kayseri, Turkey

Tel: +90-352-4372583, Fax: +90-352-4372583, E-mail: sozsoy@erciyes.edu.tr

(a) This is an Open Access article distributed under the terms of the Creative Commons Attribution Non-Commercial License (http://creativecommons.org/licenses/by$\mathrm{nc} / 3.0$ ) which permits unrestricted non-commercial use, distribution, and reproduction in any medium, provided the original work is properly cited. depressive women but not in men, and focused attention on sexual dimorphism. ${ }^{6}$ High leptin levels in women were also reported subsequently. In contrast, high leptin levels were found to be associated with an increased risk of depression onset in men in another study. ${ }^{8}$ Recent studies have focused on leptin resistance and impaired leptin action rather than changes in leptin levels in depression., ${ }^{9,10}$ The influence of antidepressant treatment on leptin levels was also investigated and controversial results were found. Reports about specific antidepressants revealed that leptin levels increased during treatment with some antidepressants such as amitriptyline and mirtazapine but no change was observed with some others such as fluoxetine, imipramine, paroxetine, and venlafaxine. ${ }^{11-13}$ Another study showed a change in leptin levels with improvement in depression. ${ }^{6}$ A study investigating the effect of ECT on leptin levels found no change in leptin levels with ECT in patients with mood disorders. ${ }^{14}$

Ghrelin, a gut hormone, has an orexigenic effect on appetite and energy balance. It is reported to be negatively associated with body fat and leptin concentration. ${ }^{15}$ Receptors for ghrelin in addition to leptin were determined in the areas of the brain responsible for regulation of emotions. In contrast 
to leptin, ghrelin was reported to be increased by acute and chronic stress in rats. ${ }^{16,17}$ While some studies suggested that administration of ghrelin revealed an increase in anxiety- and depression-like behavior in rats, ${ }^{18}$ others suggested that increasing ghrelin levels produced anxiolytic- and antidepressant-like responses in mice. ${ }^{17}$ Kluge et al. ${ }^{19}$ found that male patients showed a marginally significant decrease in depressive symptoms in being in line with an antidepressant effect of ghrelin as suggested by later study. A few human studies were performed about ghrelin levels in patients with depression and the findings were inconsistent. A study reported low ghrelin levels in depressive patients. ${ }^{20}$ However, many others suggested no difference in ghrelin levels between depressive patients and healthy controls. ${ }^{21-23}$ Nevertheless, some studies found high serum ghrelin levels in patients with major depression. ${ }^{14,24} \mathrm{~A}$ few studies about the effect of treatment of depression on ghrelin levels reported a decrease with mirtazapine, ${ }^{25}$ an increase with maprotiline, ${ }^{26}$ and a decrease with ECT. ${ }^{14}$ Previous studies about leptin and ghrelin levels in depressive patients and the effect of treatment were summarized in Table 1.

It is still not clear whether leptin and ghrelin levels are affected by depression and treatment of depression. Therefore, the present study was carried out to investigate a part of anorexigenic and orexigenic activity in depression and the effect of treatment on leptin and ghrelin levels in depressive patients. The study was designed to cross-sectionally investigate wheth-

Table 1. Studies of leptin and ghrelin in patient with depression

\begin{tabular}{|c|c|}
\hline Study & Findings \\
\hline \multicolumn{2}{|l|}{ Leptin levels in depressive patients } \\
\hline Kraus et al. $2001,{ }^{3}$ Jow et al. $2006^{4}$ & Low \\
\hline Deuschle et al. $1996^{5}$ & Unchanged \\
\hline Esel et al. $2005^{6}$ & High in depressive women but not in men \\
\hline Cizza et al. $2010^{7}$ & High nocturnal leptin levels in women \\
\hline \multicolumn{2}{|l|}{ The effect of treatment on leptin levels } \\
\hline Moosa et al. $2003^{11}$ & No change with fluoxetine, imipramine \\
\hline Schilling et al. $2013^{12}$ & $\begin{array}{l}\text { Increase with amitriptyline and mirtazapine, but not paroxetine } \\
\text { and venlafaxine }\end{array}$ \\
\hline Kraus et al. $2002^{13}$ & Increase with mirtazapine, no change with venlafaxine \\
\hline Kurt et al. $2007^{14}$ & No change with ect in patients with mood disorders \\
\hline \multicolumn{2}{|l|}{ Ghrelin levels in depressive patients } \\
\hline Barim et al. $2009^{20}$ & Low \\
\hline Schanze et al. $2008,{ }^{21}$ Kluge et al. $2009,{ }^{22}$ Matsuo et al. $2012^{23}$ & No difference \\
\hline Gecici et al. $2005^{24}$ & High \\
\hline \multicolumn{2}{|l|}{ The effect of treatment on ghrelin levels } \\
\hline Schmid et al. $2006^{25}$ & Decrease with mirtazapine \\
\hline Pinar et al. $2008^{26}$ & Increase with maprotiline \\
\hline Kurt et al. $2007^{14}$ & Decrease with ECT \\
\hline
\end{tabular}

er there is any difference between leptin and ghrelin levels of depressive patients and controls, and to longitudinally investigate the effects of improvement with antidepressant drug or ECT on leptin and ghrelin levels in depressive patients.

\section{METHODS}

\section{Subjects}

Twenty-eight patients (mean age \pm SD: $48.00 \pm 14.66$; $17 \mathrm{fe}$ male, 11 male) who met the criteria for major depressive disorder of Diagnostic and Statistical Manual of Mental Disorders, 4th edition (DSM-IV; American Psychiatric Association, 1994) were recruited for the study. The DSM-IV diagnosis of depression was determined via clinical interviews by a psychiatrist. The patients had been drug-free for at least 2 weeks. Exclusion criteria for patients were as follows: any other DSMIV disorder on the basis of clinical interviews by the psychiatrist, having a physical disease (e.g., endocrinological, metabolic) as judged from their clinical and biochemical examinations, having alcohol or drug use disorder except smoking, or receiving any hormonal therapy or oral contraceptives.

Twenty-one physically and mentally healthy controls (mean age $\pm S D: 40.85 \pm 12.39$; 11 female, 10 male) were recruited from among volunteers and hospital staff. Exclusion criteria for the controls were the same as those for the patients, plus any present or past history of psychiatric disorder. This study was car-

ECT: electroconvulsive therapy 
ried out in the Psychiatry Department of Erciyes University School of Medicine and was approved by the local Ethics Committee. Written informed consent was obtained from all patients and controls after the study had been explained to them.

\section{Procedure}

The severity of clinical symptomatology of depression was assessed using the 17-item Hamilton Depression Rating Scale (HDRS) and all patients had HDRS scores of 17 or more before the treatment. The patients were treated with ECT and/or various antidepressant drugs for 4 to 8 weeks. Eight of them were treated with ECT plus antidepressants such as venlafaxine escitalopram, and mirtazapine. These patients received a total of 7 to $10 \mathrm{ECT}$ sessions under general anesthesia, three times a week, after an overnight fast and with the electrodes placed in the bifrontotemporal position. Twenty of them received antidepressants such as venlafaxine, paroxetine, sertraline, escitalopram, and mirtazapine. The patients did not receive any non-drug therapies such as psychotherapy. A decrease of more than $50 \%$ in HDRS scores was accepted as response to the treatment. Eight patients did not complete the study.

The body mass index (BMI) was calculated by dividing the weight (kilograms) by the squared height (meters) separately before and after the treatment.

Serum levels of leptin and total ghrelin were measured before the initiation of the treatment in all patients, and after the clinical response to the treatment in those who remained in the study, and only once in the control subjects. Blood samples for leptin and ghrelin measurement were obtained at 08:0009:00 in the morning after an overnight fast. Separated serum was stored at $-70^{\circ} \mathrm{C}$ until analyzed. Serum leptin levels were determined by radioimmunoassay kits (DIAsource, Belgium). The sensitivity was $0.1 \mathrm{ng} / \mathrm{mL}$ and the intra- and inter-assay coefficients of variation were $4.4 \%$ and $5.0 \%$, respectively. Serum ghrelin levels were also determined by radioimmunoassay kits (LINCO Research, USA). The sensitivity was 7.8 pg/ $\mathrm{mL}$ and the within and between assay variations were $6.5 \%$ and $16.2 \%$, respectively.

\section{Statistical analysis}

The distributions of all variables were checked with the Shapiro-Wilk test. The patients and controls were compared with respect to sex and state of smoking using the chi-square test. The significance of differences between age, BMI, and HDRS scores of the patients and controls was assessed with the independent samples t-test, amount and duration of smoking was assessed with the Mann Whitney-U test. To compare ghrelin levels of the patients and controls the univariate-ANCOVA test was performed by taking the presence of disorder as a fixed factor, and age and BMI as covariates. The analyses of subgroups according to type of treatment (drugs and ECT), sex and severity of depression (mild to moderate vs. severe depression $\geq 28$ ) were performed using the same tests for comparison of ghrelin levels. The paired samples t-test was carried out for comparison of the clinical variables and ghrelin levels of the patients before and after the treatment. Power analyses of tests was performed for results of statistically significant differences $(\mathrm{p}<0.05)$. Nonparametric Mann Whitney-U and Wilcoxon tests were used for analyses of leptin levels, because of the abnormal distribution of leptin levels. To control the effect of age and BMI on leptin, adjusted leptin values were calculated with the formula taking BMI and age into account. The Spearman's correlation test was performed to investigate the relationships between leptin and ghrelin levels and clinical and demographical variables in the patients and controls separately.

\section{RESULTS}

The patients and controls did not differ significantly with regard to sex, age, BMI, or smoking variables. Serum ghrelin levels were significantly higher in the patients before the treatment than those of the controls ( $\mathrm{F}=4.554, \mathrm{p}=0.038$ ). There was no significant difference between leptin levels of the patients and controls (Table 2).

After the treatment, ghrelin levels decreased insignificantly $(\mathrm{p}=0.064)$ and became no different from those of the controls $(\mathrm{p}=0.415)$. The treatment did not alter the leptin levels. BMI of the patients increased after the treatment. HDRS scores of the patients were decreased by treatment but remained higher than those of the controls (Table 3).

When the effect of the treatment on hormone levels was analyzed separately in the patients treated with antidepressant drugs and those treated with ECT, no significant difference was found in terms of leptin or ghrelin levels. BMI did not change in the ECT group (Mean \pm SD: $26.52 \pm 7.82$ for pretreatment, $28.29 \pm 6.87$ for post-treatment; $\mathrm{n}=8, \mathrm{t}=1.904, \mathrm{p}=$ 0.099 ), while BMI was increased with antidepressant treatment (Mean \pm SD: $26.08 \pm 3.33$ for pre-treatment, $27.42 \pm 3.68$ for post-treatment; $\mathrm{n}=12, \mathrm{t}=2.784, \mathrm{p}=0.018$ ).

The hormone level differences between the patients and controls were also separately investigated in women and men. No significant difference was found in terms of leptin or ghrelin levels in both women $(\mathrm{Z}=0.306, \mathrm{p}=0.760 ; \mathrm{F}=1.515, \mathrm{p}=0.234$ for pre-treatment; $Z=1.215, p=0.224 ; F=0.094, p=0.762$; for post-treatment, respectively) and men $(\mathrm{Z}=0.634, \mathrm{p}=0.526 ; \mathrm{F}=$ $0.273, \mathrm{p}=0.609$ for pre-treatment; $\mathrm{Z}=0.000, \mathrm{p}=1.000 ; \mathrm{F}=0.414$, $\mathrm{p}=0.530$ for post-treatment respectively).

Patients were divided into two groups [mild to moderate $(n=13)$ vs. severe depression $(n=15)$ ] based on pre-treatment 
Table 2. Demographical and clinical characteristics and hormone levels of the patients pre-treatment and the controls

\begin{tabular}{|c|c|c|c|}
\hline & Patients $(\mathrm{N}=28)$ & Controls $(\mathrm{N}=21)$ & Comparisons \\
\hline Age $($ mean \pm SD) & $48.00 \pm 14.66$ & $40.86 \pm 12.39$ & $\mathrm{t}=1.800, \mathrm{p}=0.078$ \\
\hline Female/Male & $17 / 11$ & $11 / 10$ & $\chi^{2}=0.340, p=0.560$ \\
\hline $\mathrm{BMI}\left(\mathrm{kg} / \mathrm{m}^{2}\right)(\operatorname{mean} \pm \mathrm{SD})$ & $26.21 \pm 5.03$ & $27.27 \pm 5.58$ & $\mathrm{t}=0.695, \mathrm{p}=0.491$ \\
\hline Number of smoker & 15 & 6 & $\chi^{2}=3.063, p=0.080$ \\
\hline Cigarette consumption (number/day) [median (IR)] & $20(0)$ & $20(12.5)$ & $\mathrm{Z}=0.268, \mathrm{p}=0.789$ \\
\hline Duration of smoking (year) [median (IR)] & $20(5)$ & $17(9.5)$ & $\mathrm{Z}=0.701, \mathrm{p}=0.483$ \\
\hline HDRS score (mean \pm SD) & $27.67 \pm 7.05^{*}$ & $1.67 \pm 1.59$ & $\mathrm{t}=18.878, \mathrm{p}<0.001^{\dagger}$ \\
\hline Leptin level (ng/mL) [median (IR)] & $9.84(15.19)$ & $8.46(9.71)$ & \\
\hline Adjusted median (IR) & $7.29(20.84)$ & $8.43(13.90)$ & $\mathrm{Z}=0.040, \mathrm{p}=0.968$ \\
\hline Ghrelin level (pg/mL) (mean \pm SD) & $417.23 \pm 155.67^{*}$ & $300.58 \pm 122.71$ & \\
\hline Adjusted mean \pm SE & $405.07 \pm 26.42$ & $316.79 \pm 30.69$ & $\mathrm{~F}=4.554, \mathrm{p}=0.038^{\ddagger}$ \\
\hline
\end{tabular}

*higher than those of the controls, ${ }^{\dagger}$ power of the analysis: $100 \%$, ${ }^{\ddagger}$ power of the analysis: $55.1 \%$. BMI: body mass index, HDRS: Hamilton Depression Rating Scale, IR: interquartile range, SD: standard deviation, SE: standard error

Table 3. Clinical characteristics and hormone levels of the patients pre- and post-treatment and the controls

\begin{tabular}{|c|c|c|c|c|c|}
\hline & \multicolumn{2}{|c|}{ Patients } & \multirow{2}{*}{$\begin{array}{c}\text { Controls } \\
(\mathrm{N}=21) \\
\text { Mean } \pm \mathrm{SD}\end{array}$} & \multicolumn{2}{|c|}{ Comparisons } \\
\hline & $\begin{array}{c}\text { Pre-treatment }(\mathrm{N}=20) \\
\text { Mean } \pm \mathrm{SD}\end{array}$ & $\begin{array}{c}\text { Post-treatment }(\mathrm{N}=20) \\
\text { Mean } \pm \mathrm{SD}\end{array}$ & & $\begin{array}{l}\text { Pre-treatment vs. } \\
\text { post-treatment }\end{array}$ & $\begin{array}{l}\text { Post-treatment vs. } \\
\text { controls }\end{array}$ \\
\hline$\overline{\mathrm{BMI}}\left(\mathrm{kg} / \mathrm{m}^{2}\right)$ & $26.26 \pm 5.39$ & $27.77 \pm 5.04^{*}$ & $27.27 \pm 5.58$ & $\mathrm{t}=3.295, \mathrm{p}=0.004^{\ddagger}$ & $\mathrm{t}=0.300, \mathrm{p}=0.766$ \\
\hline HDRS score & $28.90 \pm 5.98$ & $8.20 \pm 8.85^{\dagger}$ & $1.67 \pm 1.59$ & $\mathrm{t}=15.120, \mathrm{p}<0.001^{\S}$ & $\mathrm{t}=3.251, \mathrm{p}=0.004^{\pi}$ \\
\hline $\begin{array}{l}\text { Leptin level (ng/mL) } \\
\text { adjusted median (IR) }\end{array}$ & $7.29(20.21)$ & $10.11(21.15)$ & $8.43(13.90)$ & $\mathrm{Z}=1.381, \mathrm{p}=0.167$ & $\mathrm{Z}=1.043, \mathrm{p}=0.297$ \\
\hline Ghrelin level (pg/mL) & $422.16 \pm 162.61$ & $335.50 \pm 99.46$ & $300.58 \pm 122.71$ & $\mathrm{t}=1.968, \mathrm{p}=0.064$ & $\mathrm{~F}=0.678, \mathrm{p}=0.415$ \\
\hline
\end{tabular}

*higher than those in pre-treatment, tlower than those in pre-treatment and higher than those of the controls, ${ }^{ \pm}$power of the analysis: $87.9 \%$, §power of the analysis: 100\%, "power of the analysis: 87.1\%. BMI: body mass index, HDRS: Hamilton Depression Rating Scale, SD: standard deviation

HDRS scores by using cutoff scores of 28 . Pre-treatment levels of leptin and ghrelin did not differ significantly between the patients with mild to moderate and severe depression ( $\mathrm{Z}=0.852, \mathrm{p}=0.394 ; \mathrm{F}=0.931, \mathrm{p}=0.344$ respectively).

There were positive correlations between BMI and leptin levels both before and after the treatment in the patients $(\mathrm{r}=$ $0.725, \mathrm{p}<0.001 ; \mathrm{r}=0.502, \mathrm{p}=0.024$, respectively), but not in the controls $(\mathrm{r}=0.158, \mathrm{p}=0.493)$. Ghrelin levels and BMI were not correlated in the patients $(\mathrm{r}=-0.142, \mathrm{p}=0.470$ for pre-treatment, $\mathrm{r}=-0.310, \mathrm{p}=0.184$ for post-treatment) or controls ( $\mathrm{r}=$ $-0.006, p=0.978$ ). Leptin levels were correlated positively with age $(\mathrm{r}=0.575, \mathrm{p}=0.006)$ in the controls, but not in the patients. There was no correlation between leptin or ghrelin levels and illness severity (HDRS scores) in pre-treatment $(\mathrm{r}=-0.080, \mathrm{p}=$ $0.686 ; \mathrm{r}=-0.116, \mathrm{p}=0.557$, respectively) and post-treatment $(\mathrm{r}=$ $-0.237, \mathrm{p}=0.314 ; \mathrm{r}=-0.382, \mathrm{p}=0.096$, respectively).

\section{DISCUSSION}

The main finding of the present study is that serum ghrelin levels in depressive patients were higher than those in the controls and were normalized by treatment. Similar results concerning high ghrelin levels in depression have been reported in previous studies. ${ }^{14,24}$ Some other studies have found decreased or unchanged ghrelin levels in depressive patients, inconsistent with our results. ${ }^{20-23}$ Although the finding of increased serum ghrelin level is inconsistent with some previous studies, it is in agreement with data on the relation between ghrelin and stress and depression. Ghrelin levels have been shown to be increased by chronic stress and the activation of ghrelin signaling has been explained as an adaptation response to chronic stress. ${ }^{17}$ Increased ghrelin level may be a response to chronic stress produced by depression and may play a role as an endogenous antidepressant. Likewise, endogenous antidepressant effect of ghrelin has been suggested previous studies. ${ }^{17,19}$

High ghrelin levels may be associated with some depressive symptoms such as loss of weight, appetite, and energy. This relation has been supported by reports that ghrelin release was increased by restriction of food intake and energy insufficiency. ${ }^{17,27}$ Increase in ghrelin may be a response to depressive symptoms such as loss of appetite. 
Another possible explanation of the result may be that increased ghrelin levels are related to dysfunction of the HPA axis in depression. Ghrelin induces HPA axis activity by increasing cortisol, ACTH, and CRF. ${ }^{19,28,29}$ Hyperactivity of the HPA axis is one of the pathophysiological mechanisms of depression especially in melancholic depression. ${ }^{30}$ Stress induces ghrelin signaling probably by stimulating HPA axis activity. Likewise, it has been shown that stress increased ACTH and ghrelin levels. ${ }^{16}$ In addition, glucocorticoids have been demonstrated to stimulate ghrelin. ${ }^{31}$ Our finding together with these data suggests that increased ghrelin may be associated with HPA axis hyperactivity in depression. Nevertheless, we cannot propose this hypothesis with certainty, because we did not evaluate HPA axis activity in the present study.

The other important finding of the present study is that high serum ghrelin levels were normalized by treatment in depressive patients. If we had used a specific antidepressant or ECT alone, we could have examined the effect of specific treatments. Therefore, we may suggest that improvement in depression made ghrelin levels of the patients similar to those of the controls. We found a non-significant decrease in ghrelin levels with treatment. A decrease in ghrelin levels with treatment was also reported in previous studies. ${ }^{14,25}$ Normalization of ghrelin levels with treatment may be associated with normalization of HPA activity. ${ }^{25}$ In addition, improvement of depressive symptoms such as loss of appetite and weight may contribute to normalization of ghrelin levels.

Weight gain frequently accompanies antidepressant treatment. Some previous studies have demonstrated decreases in ghrelin levels and increases in leptin levels with some antidepressant treatment and discussed whether these changes were related to weight gain and increasing BMI. ${ }^{11-13,25}$ Leptin, an anorexigenic hormone, and ghrelin, an orexigenic hormone, have been hypothesized to have opposing effects on the regulation of body weight. It has been suggested that ghrelin is negatively associated with body fat and leptin is positively associated with body fat. ${ }^{15,27}$ Therefore, it has been expected that weight gain with antidepressant treatment might be accompanied by decreasing ghrelin and increasing leptin levels. Nevertheless, the finding of the present study together with some previous data showed that the alteration in hormone levels, especially in leptin, could not be due to weight changes. The treatment increased BMI in the patients, but did not alter leptin levels in the present study. Likewise, a previous study has reported weight gain induced by antidepressant treatment without changed leptin levels. ${ }^{32}$ Additionally, we tried to control the confounding effect of BMI by taking as covariate both pre- and post-treatment BMI values in the statistical analysis of ghrelin levels and by calculating adjusted leptin values for pre- and post-treatment BMI values in the analysis of leptin levels. We may conclude that improvement of depression affected ghrelin levels, but not leptin levels with no relation to weight gain. Similarly, we also found that leptin levels did not differ between the patients and controls. It seems that serum ghrelin levels are associated with depression whereas leptin levels are not. Nevertheless, further studies are needed to substantiate these hypotheses. The role of leptin in depression may also be associated with leptin resistance rather than alteration in leptin levels.

The study has several limitations that must be taken into account. Main limitation is the small sample size, especially for subgroups. The analyses of subgroups according to type of treatment, sex and symptom severity revealed no significant hormone level changes. It might have caused a type II statistical error. However, these sub-analyses may give some ideas for future studies. Further studies with a larger number of subjects are needed specifically focusing on sex differences, type of treatment, and severity of depression. Another limitation is that controls and patients were not ideally matched in terms of both age and sex. Unfortunately, we were not able to interpret the effects of specific treatments, because we used multiple drugs and/or ECT. Additional limitations were; not using structural interview for depression diagnosis, and measuring hormone levels only in the morning in spite of diurnal variation of leptin and ghrelin.

In summary, the present study reveals increased serum ghrelin levels in depressed patients and normalization with improvement in depression but no alteration in leptin levels. The role of leptin and ghrelin in the pathophysiology of depression and the effect of treatment on appetite and weight regulation system have still not been clearly established and are worth investigating in further studies.

\section{REFERENCES}

1. Friedman JM. Leptin at 14 y of age: an ongoing story. Am J Clin Nutr 2009;89:973S-979S.

2. Lu XY, Kim CS, Frazer A, Zhang W. Leptin: a potential novel antidepressant. Proc Natl Acad Sci U S A 2006;103:1593-1598.

3. Kraus T, Haack M, Schuld A, Hinze-Selch D, Pollmacher T. Low leptin levels but normal body mass indices in patients with depression or schizophrenia. Neuroendocrinology 2001;73:243-247.

4. Jow GM, Yang TT, Chen CL. Leptin and cholesterol levels are low in major depressive disorder, but high in schizophrenia. J Affect Disord 2006;90:21-27.

5. Deuschle M, Blum WF, Englaro P, Schweiger U, Weber B, Pflaum CD, et al. Plasma leptin in depressed patients and healthy controls. Horm Metab Res 1996;28:714-717.

6. Esel E, Ozsoy S, Tutus A, Sofuoglu S, Kartalci S, Bayram F, et al. Effects of antidepressant treatment and of gender on serum leptin levels in patients with major depression. Prog Neuropsychopharmacol Biol Psychiatry 2005;29:565-570.

7. Cizza G, Nguyen VT, Eskandari F, Duan Z, Wright EC, Reynolds JC, et al. Low 24-hour adiponectin and high nocturnal leptin concentrations in a case-control study of community-dwelling premenopausal 
women with major depressive disorder: the Premenopausal, Osteopenia/Osteoporosis, Women, Alendronate, Depression (POWER) study. J Clin Psychiatry 2010;71:1079-1087.

8. Milaneschi Y, Simonsick EM, Vogelzangs N, Strotmeyer ES, Yaffe K, Harris TB, et al. Leptin, abdominal obesity, and onset of depression in older men and women. J Clin Psychiatry 2012;73:1205-1211.

9. Yamada N, Katsuura G, Ochi Y, Ebihara K, Kusakabe T, Hosoda K, et al. Impaired CNS leptin action is implicated in depression associated with obesity. Endocrinology 2011;152:2634-2643.

10. Lu XY. The leptin hypothesis of depression: a potential link between mood disorders and obesity? Curr Opin Pharmacol 2007;7:648-652.

11. Moosa MY, Panz VR, Jeenah FY, Joffe BI. African women with depression: the effect of imipramine and fluoxetine on body mass index and leptin secretion. J Clin Psychopharmacol 2003;23:549-552.

12. Schilling C, Gilles M, Blum WF, Daseking E, Colla M, Weber-Hamann $\mathrm{B}$, et al. Leptin plasma concentrations increase during antidepressant treatment with amitriptyline and mirtazapine, but not paroxetine and venlafaxine: leptin resistance mediated by antihistaminergic activity? J Clin Psychopharmacol 2013;33:99-103.

13. Kraus T, Haack M, Schuld A, Hinze-Selch D, Koethe D, Pollmacher T. Body weight, the tumor necrosis factor system, and leptin production during treatment with mirtazapine or venlafaxine. Pharmacopsychiatry 2002;35:220-225.

14. Kurt E, Guler O, Serteser M, Cansel N, Ozbulut O, Altinbas K, et al. The effects of electroconvulsive therapy on ghrelin, leptin and cholesterol levels in patients with mood disorders. Neurosci Lett 2007;426:49-53.

15. Tschop M, Weyer C, Tataranni PA, Devanarayan V, Ravussin E, Heiman ML. Circulating ghrelin levels are decreased in human obesity. Diabetes 2001;50:707-709.

16. Kristenssson E, Sundqvist M, Astin M, Kjerling M, Mattsson H, Dornonville de la Cour C, et al. Acute psychological stress raises plasma ghrelin in the rat. Regul Pept 2006;134:114-117.

17. Lutter M, Sakata I, Osborne-Lawrence S, Rovinsky SA, Anderson JG, Jung S, et al. The orexigenic hormone ghrelin defends against depressive symptoms of chronic stress. Nat Neurosci 2008;11:752-753.

18. Hansson C, Haage D, Taube M, Egecioglu E, Salome N, Dickson SL. Central administration of ghrelin alters emotional responses in rats: behavioural, electrophysiological and molecular evidence. Neuroscience 2011;180:201-211.

19. Kluge M, Schussler P, Dresler M, Schmidt D, Yassouridis A, Uhr M, et al. Effects of ghrelin on psychopathology, sleep and secretion of cortisol and growth hormone in patients with major depression. J Psychiatr Res 2011;45:421-426.

20. Barim AO, Aydin S, Colak R, Dag E, Deniz O, Sahin I. Ghrelin, paraoxonase and arylesterase levels in depressive patients before and after citalopram treatment. Clin Biochem 2009;42:1076-1081.

21. Schanze A, Reulbach U, Scheuchenzuber M, Groschl M, Kornhuber J, Kraus T. Ghrelin and eating disturbances in psychiatric disorders. Neuropsychobiology 2008;57:126-130.

22. Kluge M, Schussler P, Schmid D, Uhr M, Kleyer S, Yassouridis A, et al. Ghrelin plasma levels are not altered in major depression. Neuropsychobiology 2009;59:199-204.

23. Matsuo K, Nakano M, Nakashima M, Watanuki T, Egashira K, Matsubara T, et al. Neural correlates of plasma acylated ghrelin level in individuals with major depressive disorder. Brain Res 2012;1473:185-192.

24. Gecici O, Serteser M, Emul M, Demirel R. Serum ghrelin and leptin levels in major depressive disorders. Neurol Psychiatry Brain Res 2005; 12:47-52.

25. Schmid DA, Wichniak A, Uhr M, Ising M, Brunner $\mathrm{H}$, Held K, et al. Changes of sleep architecture, spectral composition of sleep EEG, the nocturnal secretion of cortisol, ACTH, GH, prolactin, melatonin, ghrelin, and leptin, and the DEX-CRH test in depressed patients during treatment with mirtazapine. Neuropsychopharmacology 2006;31: 832-844.

26. Pinar M, Gulsun M, Tasci I, Erdil A, Bolu E, Acikel C, et al. Maprotiline induced weight gain in depressive disorder: changes in circulating ghrelin and adiponectin levels and insulin sensitivity. Prog Neuropsychopharmacol Biol Psychiatry 2008;32:135-139.

27. Zigman JM, Elmquist JK. Minireview: From anorexia to obesity--the yin and yang of body weight control. Endocrinology 2003;144:37493756.

28. Schmid DA, Held K, Ising M, Uhr M, Weikel JC, Steiger A. Ghrelin stimulates appetite, imagination of food, GH, ACTH, and cortisol, but does not affect leptin in normal controls. Neuropsychopharmacology 2005;30:1187-1192.

29. Kageyama K, Kumata Y, Akimoto K, Takayasu S, Tamasawa N, Suda T. Ghrelin stimulates corticotropin-releasing factor and vasopressin gene expression in rat hypothalamic 4B cells. Stress 2011;14:520-529.

30. Lamers F, Vogelzangs N, Merikangas KR, de Jonge P, Beekman AT, Penninx BW. Evidence for a differential role of HPA-axis function, inflammation and metabolic syndrome in melancholic versus atypical depression. Mol Psychiatry 2013;18:692-699.

31. Kageyama K, Akimoto K, Yamagata S, Sugiyama A, Murasawa S, Watanuki $Y$, et al. Dexamethasone stimulates the expression of ghrelin and its receptor in rat hypothalamic 4B cells. Regul Pept 2012;174:12-17.

32. Hinze-Selch D, Schuld A, Kraus T, Kuhn M, Uhr M, Haack M, et al. Effects of antidepressants on weight and on the plasma levels of leptin, TNF-alpha and soluble TNF receptors: A longitudinal study in patients treated with amitriptyline or paroxetine. Neuropsychopharmacology 2000;23:13-19. 